\title{
High-intensity interval training: Modulating interval duration in overweight/obese men
}

\author{
Abbie E. Smith-Ryan, Malia N. Melvin, and Hailee L. Wingfield \\ Applied Physiology Laboratory, Department of Exercise and Sport Science, University of North \\ Carolina, Chapel Hill, NC, USA
}

\begin{abstract}
Introduction-High-intensity interval training (HIIT) is a time-efficient strategy shown to induce various cardiovascular and metabolic adaptations. Little is known about the optimal tolerable combination of intensity and volume necessary for adaptations, especially in clinical populations.
\end{abstract}

Objectives-In a randomized controlled pilot design, we evaluated the effects of two types of interval training protocols, varying in intensity and interval duration, on clinical outcomes in overweight/obese men.

\begin{abstract}
Methods-Twenty-five men [body mass index (BMI) $>25 \mathrm{~kg} \cdot \mathrm{m}^{2}$ ] completed baseline body composition measures: fat mass (FM), lean mass (LM) and percent body fat (\%BF) and fasting blood glucose, lipids and insulin (IN). A graded exercise cycling test was completed for peak oxygen consumption $\left(\mathrm{VO}_{2}\right.$ peak) and power output $(\mathrm{PO})$. Participants were randomly assigned to high-intensity short interval (1MIN-HIIT), high-intensity interval (2MIN-HIIT) or control groups. $1 \mathrm{MIN}-\mathrm{HIIT}$ and 2MIN-HIIT completed 3 weeks of cycling interval training, 3 days/week, consisting of either $10 \times 1 \mathrm{~min}$ bouts at $90 \%$ PO with $1 \mathrm{~min}$ rests (1MIN-HIIT) or $5 \times 2$ min bouts with $1 \mathrm{~min}$ rests at undulating intensities $(80 \%-100 \%)$ (2MIN-HIIT).
\end{abstract}

Results-There were no significant training effects on FM $(\Delta 1.06 \pm 1.25 \mathrm{~kg})$ or $\% \mathrm{BF}(\Delta 1.13 \% \pm$ $1.88 \%$ ), compared to CON. Increases in LM were not significant but increased by $1.7 \mathrm{~kg}$ and 2.1 $\mathrm{kg}$ for $1 \mathrm{MIN}$ and 2MIN-HIIT groups, respectively. Increases in $\mathrm{VO}_{2}$ peak were also not significant for $1 \mathrm{MIN}\left(3.4 \mathrm{ml} \cdot \mathrm{kg}^{-1} \cdot \mathrm{min}^{-1}\right)$ or $2 \mathrm{MIN}$ groups $\left(2.7 \mathrm{ml} \cdot \mathrm{kg}^{-1} \cdot \mathrm{min}^{-1}\right)$. IN sensitivity (HOMA-IR) improved for both training groups $(\Delta-2.78 \pm 3.48$ units; $p<0.05)$ compared to CON.

Conclusion-HIIT may be an effective short-term strategy to improve cardiorespiratory fitness and IN sensitivity in overweight males.

\section{Keywords}

Exercise; body weight; percent body fat; lifestyle; insulin; metabolism; $\mathrm{VO}_{2}$ peak

\footnotetext{
(C) 2015 Informa UK Ltd.

Correspondence: Abbie E. Smith-Ryan, Applied Physiology Laboratory, Department of Exercise and Sport Science, University of North Carolina, 201 Fetzer Hall, CB\#8700, Chapel Hill, NC 27599, USA. abbsmith@email.unc.edu.

Declaration of interest

The authors have no relevant affiliations or financial involvement with any organization or entity with a financial interest in or financial conflict with the subject matter or materials discussed in the manuscript.
} 


\section{Introduction}

The prevalence of obesity is well known with $68.2 \%$ of US adults falling into an overweight and obese classification [1]. More notable concerns are directly related to comorbidities such as diabetes mellitus, hypertension and cardiovascular disease. The benefit of exercise, independent of weight loss, is a known strategy to improve health and mitigate consequences of these comorbidities [2]. The most predictive factor for developing cardiovascular disease has been to be a result of low cardiorespiratory fitness (CRF) [3], which is one of the most modifiable strategies for primary and secondary prevention of cardiovascular disease [3]. While improvements in CRF are supported for health and disease, lack of time is cited as one of the most common barriers to exercise [4].

Moderate-intensity steady-state activity is promoted as the most effective way to improve body composition and CRF [5]. High-intensity interval training (HIIT) has gained attention as a time-efficient and effective method for improving body composition and augmenting cardiorespiratory health in a variety of populations, including the obese [6,7]. HIIT has been shown to stimulate a number of skeletal muscle adaptations that augment fat oxidation and oxygen utilization [6]. As a result of enhanced mitochondrial biogenesis and upregulated enzymes, a number of metabolic parameters are improved following interval training [7].

Weight regain and fat deposition is highly correlated with poor blood glucose levels, insulin (IN) resistance and high body fat [8]. HIIT has been shown to be a potent stimulus for improvements in these metabolic parameters, as well as a potential increase in excess postexercise oxygen consumption [9]. When assessing health risks, well-established blood markers, such as fasting blood glucose, total cholesterol (TC), triglycerides (TG), low (LDL) and high-density lipoproteins (HDL) and IN resistance have been shown to be reliable indicators of metabolic risk [10]. Previous studies have shown as little as 2 weeks of interval training can elicit improvements in blood glucose in a type 2 diabetic group [11], whereas Skleryk et al. [12] demonstrated no effect on metabolic function. Interval training has also been shown to be an effective method to improve cholesterol $[13,14]$ and IN sensitivity $[15,16]$. Further research is needed to establish the potential effects of acute interval training on metabolic function.

The feasibility of implementing HIIT into an overweight and obese population, where capacity and potentially mobility are reduced, is critical. One potential barrier is the intensity of the HIIT program [7,17]. While there is data to suggest benefit from all-out 30-s bouts of HIIT, this may not be the most feasible for an overweight cohort. Extending the duration beyond $30 \mathrm{~s}$, and subsequently reduction in intensity, has been suggested to threaten the effectiveness of HIIT training [18]. However, recent data have suggested these longer intervals may be beneficial for health outcomes $[11,12,16]$. Therefore, the purpose of this study was to compare the effects of two types of HIIT programs with longer interval duration, and varied intensity, in overweight/obese men on various clinical outcomes. We hypothesized that both a shorter duration interval length program (short interval training (1MIN-HIIT); 1 min bouts) and a longer duration interval length (high-intensity training; 
(1MIN-HIIT); 2 min bouts) would induce significant improvements compared to control, with no between group differences.

\section{Materials and methods}

This was a randomized controlled pilot trial. Participants were recruited from an Urban Southeast region in the US using e-mail and flyer recruitment materials. Following initial email and telephone screening, 35 men completed an in-person screening and eligibility visit. Participants were included if they were between the ages of 18 and 50 years, had a body mass index (BMI) between 25 and $45 \mathrm{~kg} \cdot \mathrm{m}^{2}$, a normal resting 12-lead electrocardiogram (EKG), and were approved by their physician. Exclusion criteria included untreated hypertension, hyperlipidemia and diabetes; previous disease history of cardiopulmonary or cardiac-related diseases, or current participation in high-intensity exercise. If potential participants had been taking anti-hypertensive, anti-depressants or lipid-lowering medications for more than 1-year, they were allowed into the study. Ten potential participants were excluded for reasons including: BMI $<25 \mathrm{~kg} \cdot \mathrm{m}^{2}$, lack of correspondence from physician (assuming no clearance) or current participation in high-intensity exercise. At the screening/enrollment visit (visit 1), all participants provided a written informed consent approved by the University Biomedical Institutional Review Board, completed a medical history questionnaire and a 12-lead EKG. Informed consent was obtained from all individual participants included in the study, and all procedures were completed in accordance with the ethical standards of the Declaration of Helsinki (2013). Twenty-five overweight men were cleared to participate in this study (mean $\pm \mathrm{SD}$; age: $38.3 \pm 11.5$ years; height: $181.9 \pm 7.5 \mathrm{~cm}$; body mass: $103.3 \pm 16.8 \mathrm{~kg}$; BMI: $31.3 \pm 4.9 \mathrm{~kg} \cdot \mathrm{m}^{2}$ ). The sample consisted of equal Caucasian and African American males, with one Asian. Eligible participants completed baseline assessments for body composition (visit 2), blood lipids (visit 2) and CRF (visit 3) (Figure 1). Prior to all testing, participants were asked to refrain from caffeine and exercise for $24 \mathrm{~h}$. Following baseline testing, participants were randomly assigned to one of two training interventions, or a control group (no training). The allocation ratio was set at 2:2:1 for the two training intervention and control groups, respectively. The group was randomly assigned using Random Allocation Software (Version 1.0.0, Isfahan, Iran). Within 1 week of randomization, participants completed day one of their nine supervised training sessions, conducted 3 days/week for 3 weeks. Post-testing assessments (visits 13-14) occurred between 24 and $48 \mathrm{~h}$ after their last training session. No adverse events were reported, and compliance was $100 \%$.

\section{Body composition assessments}

Following an 8-h fast, dual energy X-ray absorptiometry (DXA, Hologic Discovery W, Bedford, MA) was used to measure whole body composition (Apex Software Version 3.3). Using a rectilinear fan beam, whole body fat mass (FM; kg), lean mass (LM; $\mathrm{kg}$ ) and percent body fat (\%BF; \%) were measured. After removing all metal objects, participants were positioned in the center of the platform, supine, with hands facedown near their sides. If necessary, for width constraints, thumbs were slid under their legs. Participants were instructed to remain still and breathe normal for the duration of the 6-min scan. All scans were performed and analyzed by the same individual. The device was calibrated prior to 
each use according to the manufacturer to ensure valid results. Testretest reliability, in a similar population, from our lab for DXA were FM: intra-class correlation (ICC) $=0.98$, standard error of the mean $(\mathrm{SEM})=0.85 \mathrm{~kg} ; \mathrm{LM}: \mathrm{ICC}=0.99, \mathrm{SEM}=1.07 \mathrm{~kg} ; \% \mathrm{BF}: \mathrm{ICC}=$ $0.98, \mathrm{SEM}=1.06 \%$.

\section{Cardiorespiratory fitness}

Before and after training, participants performed a continuous graded exercise test on an electronically braked cycle ergometer (Corival 400, Groningen, The Netherlands) to determine peak oxygen consumption $\left(\mathrm{VO}_{2}\right.$ peak) and peak power output (PO). Pedal cadence was maintained at $70 \mathrm{rpm}$, while the PO was initially set at 50 watts (W) for a 5-min warmup, and increased by $1 \mathrm{~W}$ every $3 \mathrm{~s}$ until the participant could no longer maintain the PO (cadence dropped below $50 \mathrm{rpm}$ ). Respiratory gases were monitored and continuously analyzed with open-circuit spirometry using a calibrated metabolic cart (True One $2400^{\circledR}$, Parvo-Medics, Inc., Provo, UT). Data were averaged over 15-s intervals, with the highest 15-s oxygen consumption identified as the $\mathrm{VO}_{2}$ peak and the final time of completion identified as time to fatigue (TTF). The test was considered maximal if it met a minimum of two of the following criteria: a plateau in heart rate (HR) or HR within $10 \%$ of maximal predicted HR; a plateau in $\mathrm{VO}_{2}$ or an increase of no more than $150 \mathrm{ml} \cdot \mathrm{min}^{-1}$; a respiratory exchange ratio value greater than 1.15 . HR was monitored continuously during exercise by a HR monitor (Polar FS1, Polar Electro Inc. Lake Success, NY). Test-retest reliability for the $\mathrm{VO}_{2}$ peak protocol demonstrated reliable between-day testing with an ICC of 0.98 and SEM of $1.74 \mathrm{ml} \cdot \mathrm{kg}^{-1} \cdot \mathrm{min}^{-1}$.

All training was performed on an electronically braked cycle ergometer (Corival Lode, Gronigen, The Netherlands) under the supervision of trained research staff. Participants were required to train three times a week, with no more than two training sessions back to back (i.e. Mon, Tues, Thurs; Tues, Wed, Fri). There was a minimum of $24 \mathrm{~h}$ in between training sessions, and at least $48 \mathrm{~h}$ if two training sessions were completed consecutively. Respective groups consisted of: short interval training (1MIN-HIIT): 10 repetitions of $1 \mathrm{~min}$ bouts with 1 min rest periods at $90 \%$ of the $\mathrm{PO}$ obtained during $\mathrm{VO}_{2}$ peak (total of $10 \mathrm{~min}$ of cycling) [19]; high-intensity training (2MIN-HIIT): 5 bouts of 2 min cycling with $1 \mathrm{~min}$ recovery utilizing undulating intensities $\left(80 \%-100 \% \mathrm{VO}_{2}\right.$ peak; Figure 1) (modified from: $[16,20])$, or no exercise at all (CON). The $2 \mathrm{MIN}-\mathrm{HIIT}$ group alternated intensity as follows: 80\% (D1), 85\% (D2), 80\% (D3), 90\% (D4), 80\% (D5), 95\% (D6), 80\% (D7), 100\% (D8) and $80 \%$ (D9). Both training groups were equalized for training volume. HR and ratings of perceived exertion were measured and tracked to monitor intensity.

Serum blood samples were drawn at the University Hospital. All samples, excluding IN, were separated and processed by McLendon Clinical Laboratories (Chapel Hill, NC). IN was analyzed by Mayo Clinic Laboratories (Rochester MN). All samples were analyzed using established enzymatic assays for fasting blood glucose (GLU), TC, TG, HDL and IN. LDL and very low-density lipoprotein cholesterol (VLDL) were calculated using Friedwald's equations [LDL $=\mathrm{TC}-\mathrm{TG} / 2.2 ; \mathrm{VLDL}=\mathrm{TC}-(\mathrm{LDL}+\mathrm{HDL})]$. Furthermore, a homeostasis model assessment of IN resistance (HOMA-IR) was used to evaluate IN 
resistance, and calculated as follows: fasting IN [(IU/L) $\times$ fasting plasma glucose $\mathrm{mg} / \mathrm{dL}] /$ 405, as described by Matthews et al. [21].

\section{Statistical analyses}

All post-testing values were examined using an analysis of covariance with the baseline scores used as the covariate following verification of the homogeneity of regression assumption. When significant interactions occurred, Bonferroni post-hoc pairwise comparisons were made. Non-normally distributed variables (BMI, FM) were logtransformed before analysis. Descriptive statistics are presented as mean \pm SD. All statistical procedures were performed using SPSS (version 20.0, SPSS Inc., Chicago, IL). Ninety-five percent confidence intervals were constructed using the mean change from preto posttesting. Power calculations were completed using nQuery + nTerim 2.0 (Statistical Solutions, Boston, MA) based on previous data in overweight/obese population for $\mathrm{VO}_{2}$ peak with a $\mathrm{SD}$ of $2.5 \mathrm{ml} \cdot \mathrm{kg} \cdot \mathrm{min}^{-1}$, with the current planned sample providing a power above 0.80 . Significance for all statistical analyses was determined using a two-sided alpha of 0.05 .

\section{Results}

Training specific subject demographics for 2MIN-HIIT $(\mathrm{n}=10)$, 1MIN-HIIT $(\mathrm{n}=10)$, and $\mathrm{CON}(\mathrm{n}=5)$ are presented in Table 1 . While BMI was significantly different between groups at baseline $(p=0.021)$, there were not significant differences for percent body fat ( $p$ $=0.345)$ or CRF $(p=0.239)$. Post hoc power calculations for primary variables were adequately powered $\left(\mathrm{Vo}_{2}\right.$ peak, \% $\left.\mathrm{BF}\right)$. Lean body mass was slightly under powered (power $=$ $0.70)$.

\section{Body composition}

There was a significant main effect for treatment for FM $(p=0.001), \% \mathrm{BF}(p=0.001)$, and $\mathrm{LM}(p=0.001)$. When evaluating post hoc comparisons for FM, adjusting for baseline values, there was no significant difference between $2 \mathrm{MIN}-\mathrm{HIIT}$ (mean \pm SD: $28.3 \pm 0.96$ $\mathrm{kg}$ ) and $1 \mathrm{MIN}-\mathrm{HIIT}$ (mean \pm SD: $28.8 \pm 0.90 \mathrm{~kg})(p=0.374)$; and no difference between 2 MIN-HIIT and CON (mean \pm SD: $29.5 \pm 1.4 \mathrm{~kg})(p=0.144)$ or $1 \mathrm{MIN}-\mathrm{HIIT}$ and CON $(p=$ 0.370). Overall, there were negligible effects on FM (Figure $2 a$ ). For \% BF, post hoc comparisons yielded no significant differences between training groups $(p=0.633)$ when adjusting for baseline values; $2 \mathrm{MIN}-\mathrm{HITT}$ (mean \pm SD: $27.5 \% \pm 1.0 \%$ ) versus CON (mean \pm $S D: 28.8 \% \pm 1.4 \% ; p=0.145)$, or 1 MIN-HIIT (mean $\pm S D: 27.8 \% \pm 1.9 \%)$ versus CON $(p$ $=0.276$ ) (Figure $2 c$ ). For LM, post hoc comparisons yielded no significant difference between 2MIN-HIIT (mean $\pm S D: 69.5 \pm 3.4 \mathrm{~kg}$ ) and 1MIN-HIIT (mean \pm SD: $71.6 \pm 3.2$ ) $(p=0.898$ ), and no significant difference between 2MIN-HIIT and CON (mean \pm SD: 71.0 $\pm 5.4 \mathrm{~kg})(p=0.751)$ or $1 \mathrm{MIN}-\mathrm{HIIT}$ and CON $(p=0.811)$ (Figure $2 b)$. However, 2MINHIIT yielded an average $2.1 \mathrm{~kg}$ increase in LM, and 1MIN-HIIT resulted in an average 1.7 $\mathrm{kg}$ increase, compared to an average $0.4 \mathrm{~kg}$ decrease in LM for the CON, when compared to baseline values. 


\section{Cardiorespiratory fitness}

There was a significant main effect for treatment on $\mathrm{VO}_{2}$ peak $(p=0.001)$ and TTF $(p=$ 0.001). For $\mathrm{VO}_{2}$ peak, post hoc comparisons yielded no significant difference between training groups (2MIN-HIIT vs 1 MIN-HIIT mean difference $(\Delta)$ : $-0.47 \pm 2.6$ $\left.\mathrm{ml} \cdot \mathrm{kg}^{-1} \cdot \mathrm{min}^{-1} ; p=0.729\right)$ and no difference between $2 \mathrm{MIN}-\mathrm{HIIT}$ and $\mathrm{CON}(\Delta: 1.22 \pm 3.2$ $\left.\mathrm{ml} \cdot \mathrm{kg}^{-1} \cdot \mathrm{min}^{-1} ; p=0.459\right)$ or $1 \mathrm{MIN}-\mathrm{HIIT}$ and CON $\left(\Delta: 1.69 \pm 3.0 \mathrm{ml} \cdot \mathrm{kg}^{-1} \cdot \mathrm{min}^{-1} ; p=0.290\right)$ (Figure $3 a$ ). Post hoc comparisons for TTF resulted in no significant training group differences (2MIN-HIIT vs $1 \mathrm{MIN}-\mathrm{HIT}=\Delta 18.4 \pm 40.0 \mathrm{~s} ; p=0.388)$ and no differences for $2 \mathrm{MIN}$-HIIT compared to $\mathrm{CON}(\Delta 38.2 \pm 51.4 \mathrm{~s} ; p=0.152)$. There was a significant increase in TTF for 1MIN-HIIT compared to CON $(\Delta 56.6 \pm 51.4 \mathrm{~s} ; p=0.040)$ (Figure $3 b)$.

\section{Blood analyses}

Interval training had no significant effect $(p=0.076)$ on TC or TG $(p=0.898)$ (Table 2B). There was a significant treatment effect on fasting blood glucose $(p=0.009)$, HDL ( $p=$ $0.049)$, LDL $(p=0.002)$, IN $(p=0.001)$ and HOMA-IR $(p=0.001)$. The only post hoc comparisons that yielded significance were for IN and HOMA-IR $(p<0.05)$. For both variables, $2 \mathrm{MIN}-\mathrm{HIIT}$ significantly positively influenced IN (2MIN-HIIT vs CON $=\Delta$ $-12.4 \pm 8.4 \mathrm{IU} / \mathrm{L} p=0.008)$ and HOMA-IR; (2MIN-HIIT vs CON $\Delta-4.2 \pm 4.0 ; p=0.049$ ), compared to $\mathrm{CON}$. There were no significant effects for $1 \mathrm{MIN}-\mathrm{HIIT}$ when compared to CON for IN $(\Delta-7.6 \pm 8.2 \mathrm{IU} / \mathrm{L} ; p=0.079)$. However, $1 \mathrm{MIN}-\mathrm{HIIT}$ was significantly lower than CON for HOMA-IR $(\Delta-2.9 \pm 4.0 ; p=0.048)$. There were no differences between training groups.

\section{Discussion}

Traditional low-moderate intensity exercise is an efficient strategy to reduce symptoms of metabolic syndrome and improve aerobic capacity [3]; however, this type of training often does not meet time constraints. In the current pilot trial, $20 \mathrm{~min}$ of high-intensity exercise (10 min of work +10 min of rest), three times per week, yielded modest improvements for IN sensitivity, and CRF in overweight and obese men. The magnitude of improvement in the current study is similar to interventions lasting 12 or more weeks $[16,22,23]$. It is known that lack of exercise is one of the most powerful factors for chronic disease development [2]. Integrating time-efficient, and effective, exercise protocols into routines of clinical populations, may have a widespread effect on physiological health. The current results suggest that short-term high-intensity aerobic training may be an effective method to initiate lifestyle improvements in an overweight population.

Interval style exercise has previously been shown to modulate metabolism, with an increased reliance on oxidative phosphorylation after as little as six sessions [24]. Upregulating oxidative bioenergetics, and therefore enhancing fat oxidation, following exercise may indirectly improve body composition when accumulated over time [25,26]. The present study failed to induce any changes in FM (Table 2A), and yielded no significant change in percent body fat, but resulted in modest improvements in LM. In combination with enhanced mitochondrial biogenesis, muscle protein synthesis has previously been augmented following nine sessions of interval training [27]. The current study demonstrated 
non-significant increases in lean body mass from both interval duration groups ( 1 and 2 min); although not significant, an average 3-4 lb increase in LM can be considered clinically relevant [28]. Much of the previous data has evaluated 'all-out' short duration work bouts (30 s) [7,29], with more recent data evaluating longer (1-min and 4-min) duration bouts $[11,16]$, which may be feasible for clinical populations. An aim of the present study was to evaluate more feasible work durations that have shown benefit in healthy populations $[18,20]$. In the present study, both protocols elicited similar results for body composition.

Although there is a growing body of literature demonstrating an improvement in $\mathrm{VO}_{2} \mathrm{max}$ as a result of HIIT [30], the present study did not demonstrate a statistically significant improvement. A meta-analysis from Weston et al. [30]. reported a 6\%-10\% average increase in $\mathrm{VO}_{2} \max$ in sedentary males with varying interval intervention lengths and training periods (2-8 weeks). The present study demonstrated an average 5\% improvement in $\mathrm{VO}_{2}$ peak in the $2 \mathrm{MIN}-\mathrm{HIIT}$ group and $9.5 \%$ improvement in the 1MINHIIT group. So, despite the lack of significance, the improvements in the present study are in line with previous data. The lack of statistical significance may be a result of small sample size, as well as intervention duration, as the percent increase is slightly below that shown from a 12week of high-intensity training in a similar population [23]. The currently results could still have utility when there is a need for more rapid improvements in CRF, compared to more traditional aerobic training [31]. When cardiovascular fitness improvements from HIIT are compared to moderate-intensity longer duration exercise, an average $90 \%$ reduction in training volume from high-intensity training outweighs the $1 \%$ increase in $\mathrm{VO}_{2}$ max seen with lower intensity training [32]. The physiological mechanisms supporting consistent improvements, largely from sprint-interval "all-out" training, in oxygen uptake have been widely attributed to enhanced mitochondrial biogenesis, enhanced capillarization and arterial compliance, and upregulation of peroxisome prolifterator-activated receptor $\gamma$ coactivator (PGC)-1a [29,32,33]. Enhanced activation of PGC-1a has demonstrated positive effects on oxidative capacity and glucose uptake $[29,33]$.

In combination with cardiovascular effects, as few as six sessions of interval training, have demonstrated a positive effect on fasting glucose and IN sensitivity in overweight individuals [11,34]. The present study resulted in significant improvements in fasting blood glucose and IN sensitivity following three weeks interval training. Specifically, 2MIN-HIIT and 1MIN-HIIT resulted in a significant improvement in IN sensitivity (Table 2B).

Although both training protocols were matched for total volume (10 total min), a longer duration work bout, may be more efficacious for inducing positive metabolic changes (IN and HOMA-IR) in overweight men. In type II diabetics [11], short-term high-intensity training elicited positive changes in glucose control, which was hypothesized due to improved skeletal muscle glucose transport. Richards et al. [35] and Hood et al. [34] also demonstrated improvements in IN sensitivity in inactive adults following short-term interval training. Collectively, fasting glucose and IN sensitivity data from interval training are more responsive to longer duration (12+ weeks) of training [32]. The present study was unable to demonstrate any positive effects on serum lipid outcomes. A minimum duration of 8 weeks of training has been suggested as the necessary dose-response to yield improvements in these variables, specifically for HDL [32]. Other lipid variables (TC, LDL, TG) do not seem 
to be affected by interval training; instead they tend to be directly related to body composition changes [8].

\section{Conclusion}

There are limitations to all studies; this pilot trial lacks a robust control group and resulted in reduced homogeneity across groups, potentially due to randomization and relatively small sample size, and varied age of subjects. Even with these limitations, this data provides initial support for extending interval durations from an all-out $30 \mathrm{~s}$ approach, to a more manageable 1-min or 2-min bout protocol. Despite reducing the intensity, as cautioned by Boyd et al. [18], the two current training protocols appear to stimulate similar changes in CRF and IN sensitivity, compared to the shorter all-out protocols in overweight/obese men. More so, the practicality and cost of such intervention is minimal, with data demonstrating translation to home and community based effects [17]. Focusing on lifestyle improvements, integrating short duration, high-intensity training appears to be an effective strategy for enhancing health in a short period of time in overweight/obese men. More importantly, this style of exercise has minimal time demands (i.e. 20 total min) and was well tolerated by participants, with no adverse events reported and $100 \%$ compliance. Future studies should evaluate longer term (> 12 weeks) effects of these more tolerable protocols, in replace of 'all-out' maximal efforts, to evaluate the effects on chronic health concerns.

\section{Acknowledgements}

We would like to thank Mark Weaver, PhD for statistical insight. This study was funded by the Nutrition Obesity Research Center (P30DK056350). The project described was also supported by the National Center for Advancing Translational Sciences, National Institutes of Health, through Grant 1KL2TR001109. The content is solely the responsibility of the authors and does not necessarily represent the official views of the NIH.

This includes employment, consultancies, honoraria, stock ownership or options, expert testimony, grants or patents received or pending, or royalties.

\section{References}

1. Go AS, Mozaffarian D, Roger VL, Benjamin EJ, Berry JD, Borden WB, et al. Heart disease and stroke statistics-2013 update: a report from the American Heart Association. Circulation. 2013; 127:e6-e245. [PubMed: 23239837]

2. Booth FW, Roberts CK, Laye MJ. Lack of exercise is a major cause of chronic diseases. Compr Physiol. 2012; 2:1143-1211. [PubMed: 23798298]

3. Blair SN, Kohl HW 3rd, Paffenbarger RS Jr, Clark DG, Cooper KH, Gibbons LW. Physical fitness and all-cause mortality. a prospective study of healthy men and women. JAMA. 1989; 262:23952401. [PubMed: 2795824]

4. Booth FW, Gordon SE, Carlson CJ, Hamilton MT. Waging war on modern chronic diseases: primary prevention through exercise biology. J Appl Physiol. 2000; 88:774-787. [PubMed: 10658050]

5. Donnelly JE, Blair SN, Jakicic JM, Manore MM, Rankin JW, Smith BK. American college of sports medicine position stand. appropriate physical activity intervention strategies for weight loss and prevention of weight regain for adults. Med Sci Sports Exerc. 2009; 41:459-471. [PubMed: 19127177]

6. Boutcher SH. High-intensity intermittent exercise and fat loss. J Obes. 2011; 2011:868305. [PubMed: 21113312] 
7. Weston KS, Wisloff U, Coombes JS. High-intensity interval training in patients with lifestyleinduced cardiometabolic disease: a systematic review and meta-analysis. Br J Sports Med. 2014; 48:1227-1234. [PubMed: 24144531]

8. Houston MC, Fazio S, Chilton FH, Wise DE, Jones KB, Barringer TA, Bramlet DA. Nonpharmacologic treatment of dyslipidemia. Prog Cardiovasc Dis. 2009; 52:61-94. [PubMed: 19732602]

9. Larsen I, Welde B, Martins C, Tjonna AE. High- and moderate-intensity aerobic exercise and excess post-exercise oxygen consumption in men with metabolic syndrome. Scand J Med Sci Sports. 2014; 24:e174-e179. [PubMed: 24118097]

10. Racil G, Ben Ounis O, Hammouda O, Kallel A, Zouhal H, Chamari K, Amri M. Effects of high vs. moderate exercise intensity during interval training on lipids and adiponectin levels in obese young females. Eur J Appl Physiol. 2013; 113:2531-2540. [PubMed: 23824463]

11. Little JP, Gillen JB, Percival ME, Safdar A, Tarnopolsky MA, Punthakee Z, et al. Low-volume high-intensity interval training reduces hyperglycemia and increases muscle mitochondrial capacity in patients with type 2 diabetes. J Appl Physiol. 2011; 111:1554-1560. [PubMed: 21868679]

12. Skleryk JR, Karagounis LG, Hawley JA, Sharman MJ, Laursen PB, Watson G. Two weeks of reduced-volume sprint interval or traditional exercise training does not improve metabolic functioning in sedentary obese men. Diabetes Obes Metab. 2013; 15:1146-1153. [PubMed: 23802920]

13. Lamina S, Okoye GC. Therapeutic effect of a moderate intensity interval training program on the lipid profile in men with hypertension: a randomized controlled trial. Niger J Clin Pract. 2012; 15:42-47. [PubMed: 22437088]

14. Williams PT. Relationship of running intensity to hypertension, hypercholesterolemia, and diabetes. Med Sci Sports Exerc. 2008; 40:1740-1748. [PubMed: 18799983]

15. Babraj JA, Vollaard NB, Keast C, Guppy FM, Cottrell G, Timmons JA. Extremely short duration high intensity interval training substantially improves insulin action in young healthy males. BMC Endocr Disord. 2009; 9:3. [PubMed: 19175906]

16. Tjonna AE, Lee SJ, Rognmo O, Stølen TO, Bye A, Haram PM, et al. Aerobic interval training versus continuous moderate exercise as a treatment for the metabolic syndrome: a pilot study. Circulation. 2008; 118:346-354. [PubMed: 18606913]

17. Lunt H, Draper N, Marshall HC, Logan FJ, Hamlin MJ, Shearman JP, et al. High intensity interval training in a real world setting: a randomized controlled feasibility study in overweight inactive adults, measuring change in maximal oxygen uptake. PLoS ONE. 2014; 9:e83256. [PubMed: 24454698]

18. Boyd JC, Simpson CA, Jung ME, Gurd BJ. Reducing the intensity and volume of interval training diminishes cardiovascular adaptation but not mitochondrial biogenesis in overweight/obese men. PLoS One. 2013; 8:e68091. [PubMed: 23861854]

19. Gillen JB, Little JP, Punthakee Z, Tarnopolsky MA, Riddell MC, Gibala MJ. Acute high-intensity interval exercise reduces the postprandial glucose response and prevalence of hyperglycaemia in patients with type 2 diabetes. Diabetes Obes Metab. 2012; 14:575-577. [PubMed: 22268455]

20. Smith AE, Walter AA, Graef JL, Kendall KL, Moon JR, Lockwood CM, et al. Effects of betaalanine supplementation and high-intensity interval training on endurance performance and body composition in men; a double-blind trial. J Int Soc Sports Nutr. 2009; 6:5. [PubMed: 19210788]

21. Matthews DR, Hosker JP, Rudenski AS, Naylor BA, Treacher DF, Turner RC. Homeostasis model assessment: insulin resistance and beta-cell function from fasting plasma glucose and insulin concentrations in man. Diabetologia. 1985; 28:412-419. [PubMed: 3899825]

22. Helgerud J, Hoydal K, Wang E, Karlsen T, Berg P, Bjerkaas M, et al. Aerobic High-intensity intervals improve vo2max more than moderate training. Med Sci Sports Exerc. 2007; 39:665-671. [PubMed: 17414804]

23. Nybo L, Sundstrup E, Jakobsen MD, Mohr M, Hornstrup T, Simonsen L, et al. High-intensity training versus traditional exercise interventions for promoting health. Med Sci Sports Exerc. 2010; 42:1951-1958. [PubMed: 20195181] 
24. Laursen PB, Blanchard MA, Jenkins DG. Acute high-intensity interval training improves tvent and peak power output in highly trained males. Can J Appl Physiol. 2002; 27:336-348. [PubMed: 12442351]

25. De Feo P. Is high-intensity exercise better than moderate intensity exercise for weight loss? Nutr Metab Cardiovasc Dis. 2013; 23:1037-1042. [PubMed: 24119988]

26. Skelly LE, Andrews PC, Gillen JB, Martin BJ, Percival ME, Gibala MJ. High-intensity interval exercise induces 24-H energy expenditure similar to traditional endurance exercise despite reduced time commitment. Appl Physiol Nutr Metab. 2014; 39:845-848. [PubMed: 24773393]

27. Scalzo RL, Peltonen GL, Binns SE, Shankaran M, Giordano GR, Hartley DA, et al. Greater muscle protein synthesis and mitochondrial biogenesis in males compared with females during sprint interval training. FASEB J. 2014; 28:2705-2714. [PubMed: 24599968]

28. Wolfe RR. The underappreciated role of muscle in health and disease. Am J Clin Nutr. 2006; 84:475-482. [PubMed: 16960159]

29. Gibala MJ, Little JP, Macdonald MJ, Hawley JA. Physiological adaptations to low-volume, highintensity interval training in health and disease. J Physiol. 2012; 590:1077-1084. [PubMed: 22289907]

30. Weston M, Taylor KL, Batterham AM, Hopkins WG. Effects of low-volume high-intensity interval training (hit) on fitness in adults: a meta-analysis of controlled and non-controlled trials. Sports Med. 2014; 44:1005-1017. [PubMed: 24743927]

31. Laursen PB, Jenkins DG. The scientific basis for high-intensity interval training: optimising training programmes and maximising performance in highly trained endurance athletes. Sports Med. 2002; 32:53-73. [PubMed: 11772161]

32. Kessler HS, Sisson SB, Short KR. The potential for high-intensity interval training to reduce cardiometabolic disease risk. Sports Med. 2012; 42:489-509. [PubMed: 22587821]

33. Rakobowchuk M, Tanguay S, Burgomaster KA, Howarth KR, Gibala MJ, MacDonald MJ. Sprint interval and traditional endurance training induce similar improvements in peripheral arterial stiffness and flow-mediated dilation in healthy humans. Am J Physiol Regul Integr Comp Physiol. 2008; 295:R236-R242. [PubMed: 18434437]

34. Hood MS, Little JP, Tarnopolsky MA, Myslik F, Gibala MJ. Low-volume interval training improves muscle oxidative capacity in sedentary adults. Med Sci Sports Exerc. 2011; 43:18491856. [PubMed: 21448086]

35. Richards JC, Johnson TK, Kuzma JN, Lonac MC, Schweder MM, Voyles WF, Bell C. Short-term sprint interval training increases insulin sensitivity in healthy adults but does not affect the thermogenic response to beta-adrenergic stimulation. J Physiol. 2010; 588:2961-2972. [PubMed: 20547683] 


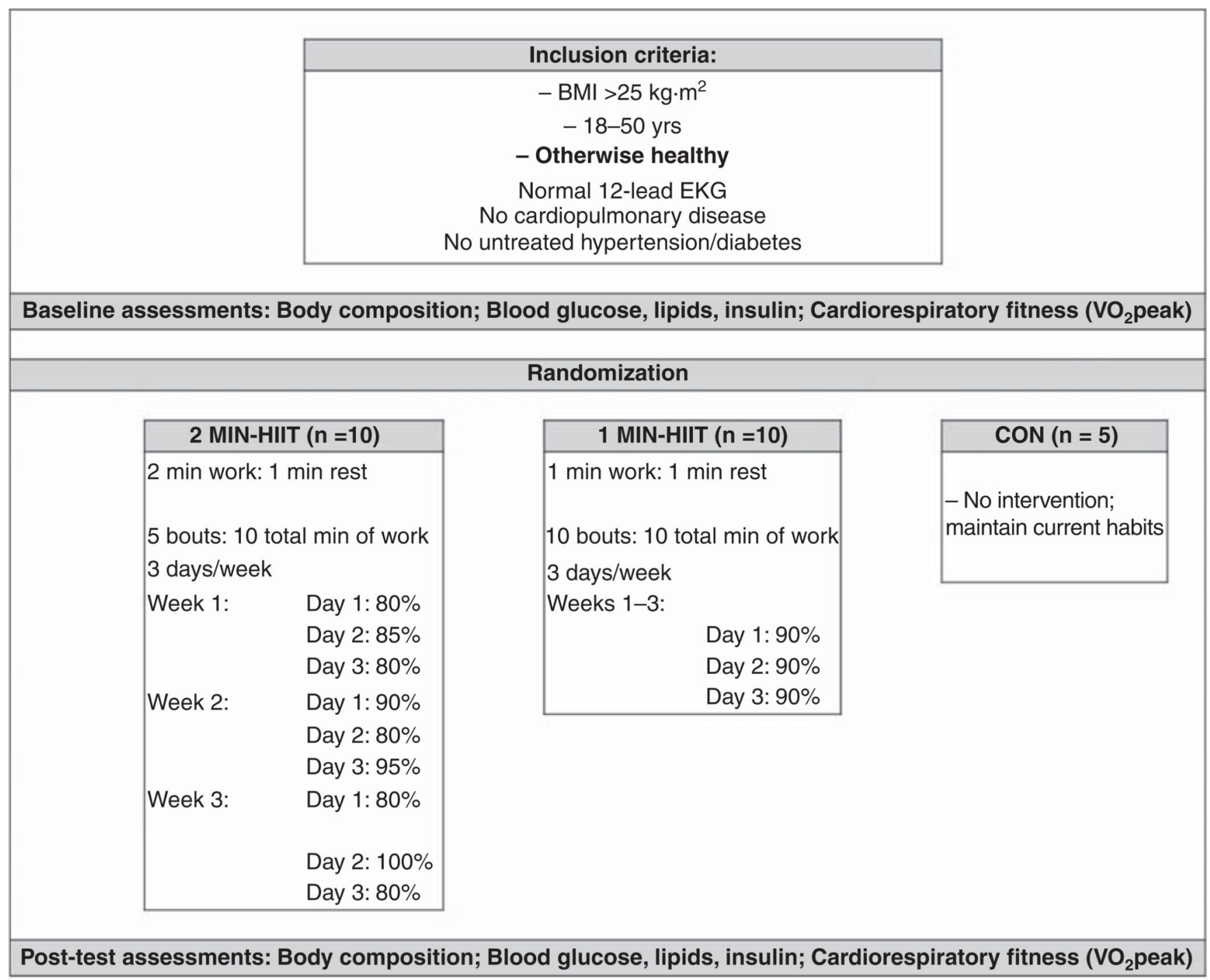

Figure 1.

Experimental design for inclusion criteria, pre-post assessments, and training protocol.

Abbreviation: HIIT $=$ High-intensity interval training. 
a
Fat mass

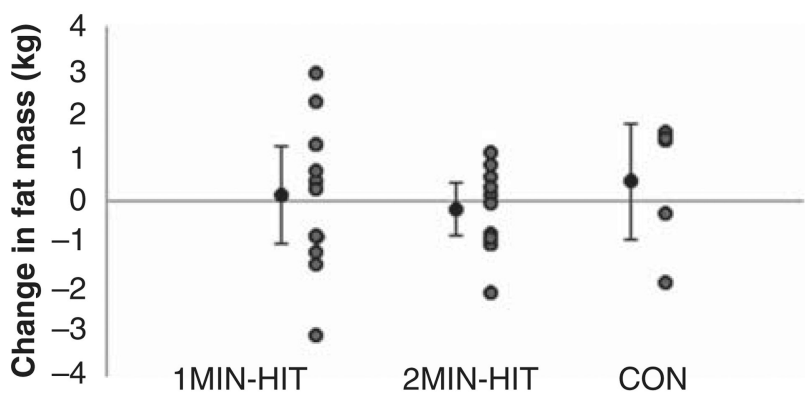

b

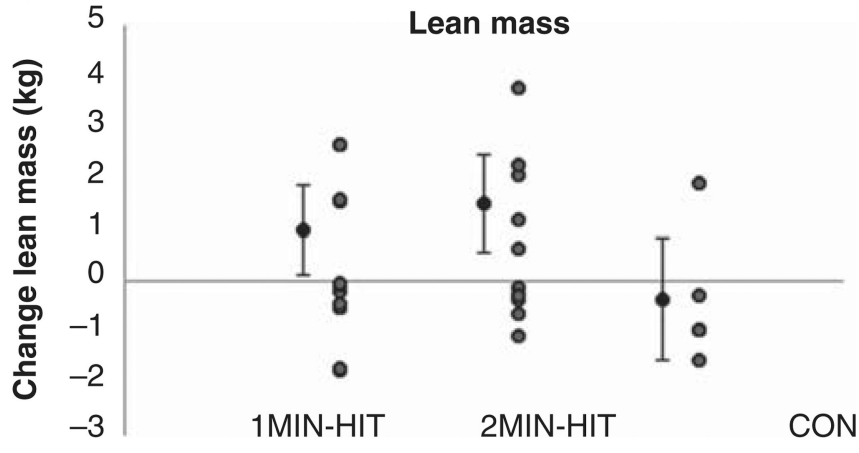

c

Percent fat

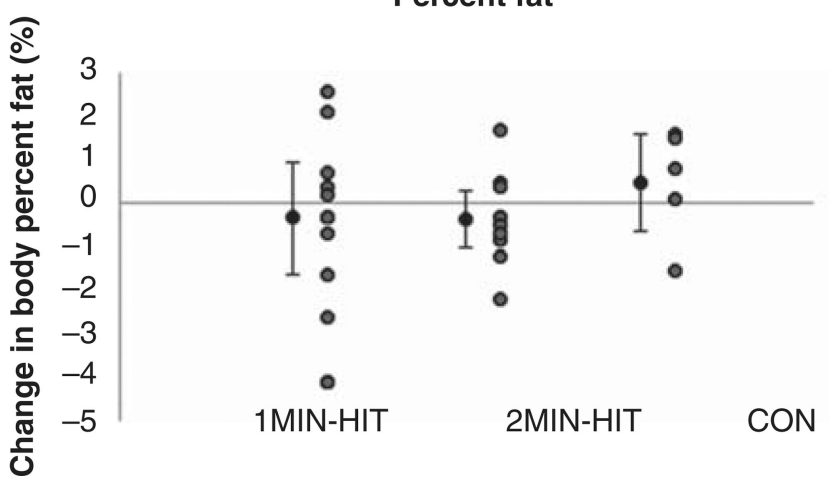

Figure 2.

Mean $\pm 95 \%$ confidence intervals and individual responses for the change in $(a)$ Fat mass $(\mathrm{kg}),(b)$ Lean mass $(\mathrm{kg})$, and $(c)$ Percent body fat (\%) for 1MIN-HIIT, 2MIN-HIIT, and CON from pre- to post-training.

Abbreviation: HIIT = High-intensity interval training. 
a

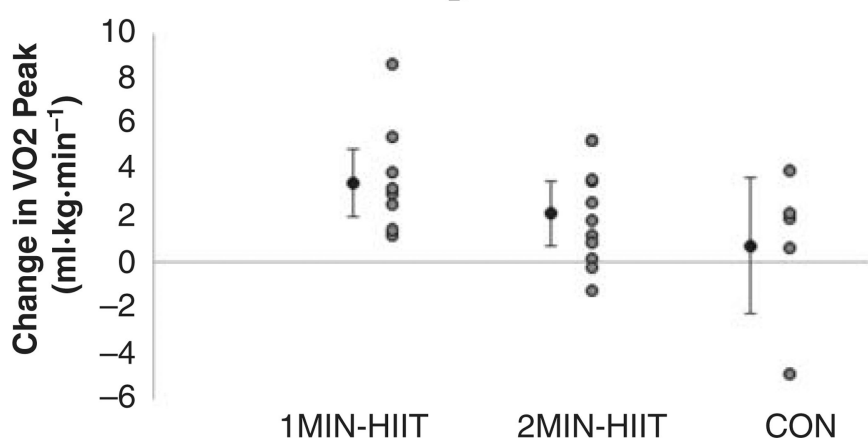

b

140

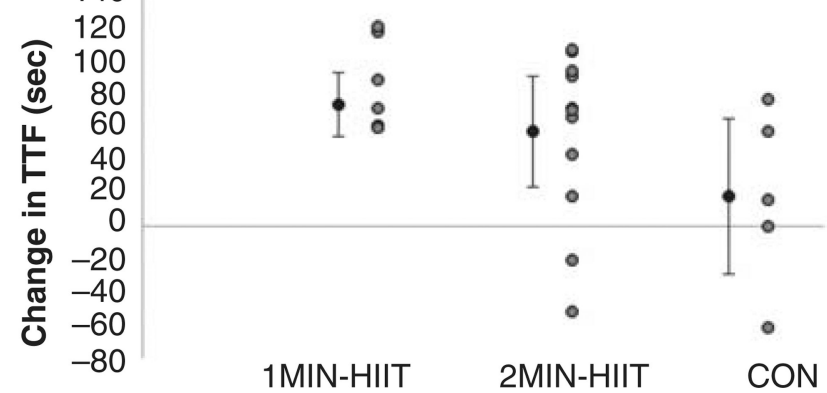

Figure 3.

Mean $\pm 95 \%$ confidence intervals and individual responses for the change in $(a) \mathrm{VO}_{2}$ peak $\left(\mathrm{ml} \cdot \mathrm{kg} \cdot \mathrm{min}^{-1}\right)$ and $(b) \mathrm{TTF}, \mathrm{s}$.

Abbreviation: $\mathrm{TTF}=$ Time to fatigue. 


\section{Table 1}

Baseline descriptive characteristics for high-intensity interval (2MIN-HIIT), short-intensity interval (1MINHIIT), and control (CON) groups.

\begin{tabular}{lcccc}
\hline & Age (years) & Height $(\mathbf{c m})$ & Weight $(\mathbf{k g})$ & BMI $\left(\mathbf{k g} \cdot \mathbf{m}^{\mathbf{2}}\right)$ \\
\hline 2MIN-HIIT $(\mathrm{n}=10)$ & $40.6 \pm 12.1$ & $181.8 \pm 9.2$ & $94.1 \pm 10.8$ & $28.4 \pm 1.3$ \\
1MIN-HIIT $(\mathrm{n}=10)$ & $36.5 \pm 12.3$ & $180.4 \pm 6.7$ & $104.0 \pm 12.3$ & $32.1 \pm 4.4$ \\
CON $(\mathrm{n}=5)$ & $37.2 \pm 9.9$ & $184.9 \pm 5.2$ & $120.5 \pm 22.6$ & $35.4 \pm 7.4^{a}$ \\
\hline
\end{tabular}

$a_{\text {Indicates significant difference between groups. }}$

Abbreviations: $\mathrm{BMI}=$ Body mass index HIIT $=$ High-intensity interval training. 


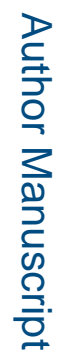

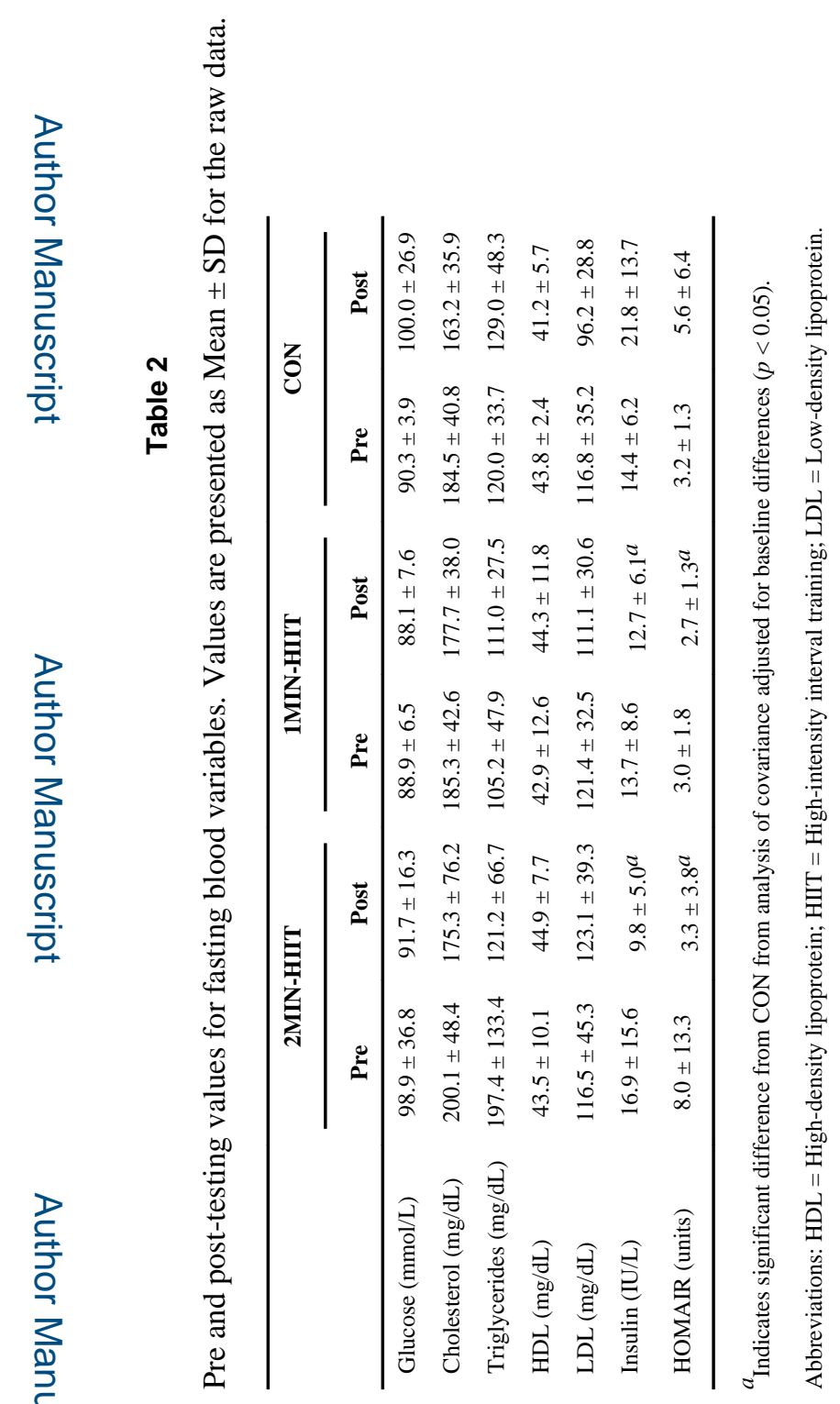

